\title{
Scattering cluster wave functions on the lattice using the adiabatic projection method
}

\author{
Alexander Rokash ${ }^{* a}$, Michelle Pine ${ }^{b}$, Serdar Elhatisari ${ }^{c b}$, Dean Lee ${ }^{b}$, Evgeny \\ Epelbaum $^{a}$ and Hermann Krebs ${ }^{a}$ \\ ${ }^{a}$ Institut für Theoretische Physik II, Ruhr-Universität Bochum, 44870 Bochum, Germany \\ ${ }^{b}$ Department of Physics, North Carolina State University, Raleigh, North Carolina 27695, USA \\ ${ }^{c}$ Helmholtz-Institut für Strahlen- und Kernphysik (Theorie) and Bethe Center for Theoretical \\ Physics, Universität Bonn, 53115 Bonn, Germany \\ E-mail: alexander.rokash@tp2.ruhr-uni-bochum.de
}

\begin{abstract}
The adiabatic projection method is a general framework for obtaining a low-energy effective Hamiltonian for clusters. Previous studies have used the adiabatic projection method in combination with the finite-volume energy Lüscher's method to extract scattering phase shifts. We discuss several methods to calculate elastic phase shifts directly from asymptotic cluster wave functions obtained from the effective cluster Hamiltonian for examples in one and three dimensions. This approach is less sensitive than the finite-volume energy Lüscher method to stochastic and systematic errors which appear in the application of the adiabatic projection method.
\end{abstract}

The 8th International Workshop on Chiral Dynamics

29 June 2015 - 03 July 2015

Pisa, Italy

\footnotetext{
* Speaker.
} 


\section{Introduction}

Recent progress in ab initio description of nuclear scattering and reactions on the lattice was achieved using different techniques [1,2]. In this report we focus on the adiabatic projection method $[3,4,5,6]$. The general strategy in this formalism is described in the pioneering works $[7,8,9]$ and involves two steps. First, an effective adiabatic Hamiltonian for the nuclei involved in the scattering process is determined using the Euclidean time projection. The original, microscopic Hamiltonian exerted in the time projection is usually derived in pionless or chiral EFT. In the second step, the corresponding scattering phase shifts are extracted [8]. We argue that we can use a method less sensitive to small stochastic and systematic errors than the usual method, namely Lüscher's finite-volume method [10, 11], for this step. The results presented in this proceeding are described in more detail in Ref. [12].

\section{The adiabatic projection method}

In the following we describe the first step of the adiabatic projection method, the derivation of the effective adiabatic Hamiltonian. In the case of a nonzero temporal lattice spacing the adiabatic Hamiltonian is replaced by an adiabatic transfer matrix. First, we define a set of two-cluster states on a $L^{3}$ periodic lattice. They are distinguished by their initial separation vector $\vec{R}$. The crucial fact about the two-cluster states is that each of the clusters is localized. Therefore for large separations they can be represented as a tensor product of two one-cluster states,

$$
|\vec{R}\rangle=\sum_{\vec{r}}|\vec{r}+\vec{R}\rangle_{1} \otimes|\vec{r}\rangle_{2}
$$

Then, Euclidean time evolution with the microscopic Hamiltonian is used in order to form dressed cluster states

$$
|\vec{R}\rangle_{\tau}=\exp (-H \tau)|\vec{R}\rangle
$$

This procedure incorporates deformations and polarizations of the interacting clusters automatically. Those dressed cluster states span the low-energy subspace of two-cluster continuum states in the limit of large Euclidean time. Finally, we evaluate the following matrices

$$
\begin{aligned}
& {\left[H_{\tau}\right]_{\vec{R}, \vec{R}^{\prime}}={ }_{\tau}\left\langle\vec{R}|H| \vec{R}^{\prime}\right\rangle_{\tau}, \quad\left[N_{\tau}\right]_{\vec{R}, \vec{R}^{\prime}}={ }_{\tau}\left\langle\vec{R} \mid \vec{R}^{\prime}\right\rangle_{\tau},} \\
& {\left[H_{\tau}^{a}\right]_{\vec{R}, \vec{R}^{\prime}}=\sum_{\vec{R}^{\prime \prime}, \vec{R}^{\prime \prime \prime}}\left[N_{\tau}^{-1 / 2}\right]_{\vec{R}, \vec{R}^{\prime \prime}}\left[H_{\tau}\right]_{\vec{R}^{\prime \prime}, \vec{R}^{\prime \prime \prime}}\left[N_{\tau}^{-1 / 2}\right]_{\vec{R}^{\prime \prime \prime}, \vec{R}^{\prime}}}
\end{aligned}
$$

Thereby, $\left[H_{\tau}\right]_{\vec{R}, \vec{R}^{\prime}}$ are matrix elements of the microscopic Hamiltonian with respect to the dressed cluster states, $N_{\tau}$ the norm matrix with respect to them and $\left[H_{\tau}^{a}\right]_{\vec{R}, \vec{R}^{\prime}}$ the adiabatic Hamiltonian matrix. The multiplication by the inverse of the norm matrix is neccesary since the dressed cluster states $|\vec{R}\rangle_{\tau}$ are, in general, not orthogonal. The square root of the norm matrix is incorporated in order to keep the adiabatic Hamiltonian symmetric.

In the limit of large Euclidean time the low-enery spectra of $H_{\tau}^{a}$ of the microscropic Hamiltonian $H$ coincide. The most evident way to do the second step of the adiabatic projection method, namely the calculation of the phase shifts, is by applying the Lüscher method to the spectrum of $H_{\tau}^{a}$. 
This method $[10,11]$ relates the energy levels of the two-body scattering states in a periodic finite volume to the same scattering solutions in the infinite volume. This method relies on the known asymptotics of the wave function for a short range interaction as well as the known periodicity of the system. The one-dimensional result of this ansatz is [10]

$$
\mathrm{e}^{2 i \delta(p)}=\mathrm{e}^{-i p L}
$$

Thereby, $p$ is the relative momentum, $L$ the size of the finite volume and $\delta(p)$ the scattering phase shift. The relative momentum $p$ is measured between the two clusters. The clusters in this context refer to either a point-like particle or a bound state of more particles. Furthermore, the total momentum of the two-cluster system is assumed to be zero. In three dimensions there is an additional complication due to the breaking of rotational invariance by the cubic symmetry of the finite periodic box. The scattering phase shifts are extracted by applying the formula $[10,11]$

$$
p \cot \delta_{\ell}(p)=\frac{1}{\pi L} S(\eta) \quad \text { for } \ell=0,1
$$

where $S(\eta)$ is a function of the relative momentum $p$ due to $\eta=\left(\frac{L p}{2 \pi}\right)^{2}$,

$$
S(\eta)=\lim _{\Lambda \rightarrow \infty}\left[\sum_{\vec{n}}^{\Lambda} \frac{\theta\left(\Lambda^{2}-\vec{n}^{2}\right)}{\vec{n}^{2}-\eta}-4 \pi \Lambda\right] .
$$

The relation between the center-of-mass momentum appearing in Eq. $(2.6,2.7)$ and the finitevolume energies for $\ell=0$ by $[13,14]$

$$
E(p, L)=\frac{p^{2}}{2 \mu}-B_{1}-B_{2}+\bar{\tau}_{1}(\eta) \Delta E_{1}(L)+\bar{\tau}_{2}(\eta) \Delta E_{2}(L),
$$

where $\mu$ is the reduced mass of the system, $B_{i}$ is the binding energy of the cluster $i=\{1,2\}$ in the infinite volume limit, $\Delta E_{i}(L)=E_{i}(L)+B_{i}$ are the finite volume energy shifts of the clusters in the rest frame, and $\bar{\tau}_{i}(\eta)$ is the topological correction factor to the energy of the cluster $i$,

$$
\bar{\tau}(\eta)=\frac{1}{\sum_{\vec{k}}\left(\vec{k}^{2}-\eta\right)^{-2}} \sum_{\vec{k}} \frac{\sum_{i=1}^{3} \cos \left(2 \pi k_{i} \alpha\right)}{3\left(\vec{k}^{2}-\eta\right)^{2}} .
$$

Lüscher's method is a very efficient tool since all of the information about scattering can be extracted from finite-volume energy values. However, the problem of that method arises due its reliance on the energy spectrum. The binding energies of scattering nuclei can be much larger than the finite-volume scattering energy shifts which encode the scattering information. Thus, these finite-volume energy shifts are very sensitive to imprecise 'measurements'. It should be measured with the precision of approximately $10 \mathrm{keV}$ in order to extract the $s$-wave scattering phase shift with the accuracy of a few degrees. However, the stochastic errors usual for a typical large-scale Monte Carlo calculation tend to violate this requirement since they grow exponentially for a large projection time $\tau$. We compare in Fig. 1 the original, microscopic Hamiltonian and the adiabatic Hamiltonian for the one dimensional particle-dimer system for $\tau=0.30 \mathrm{MeV}^{-1}$. In the left panel we show their energy spectra and in the right panel the corresponding particle-dimer 

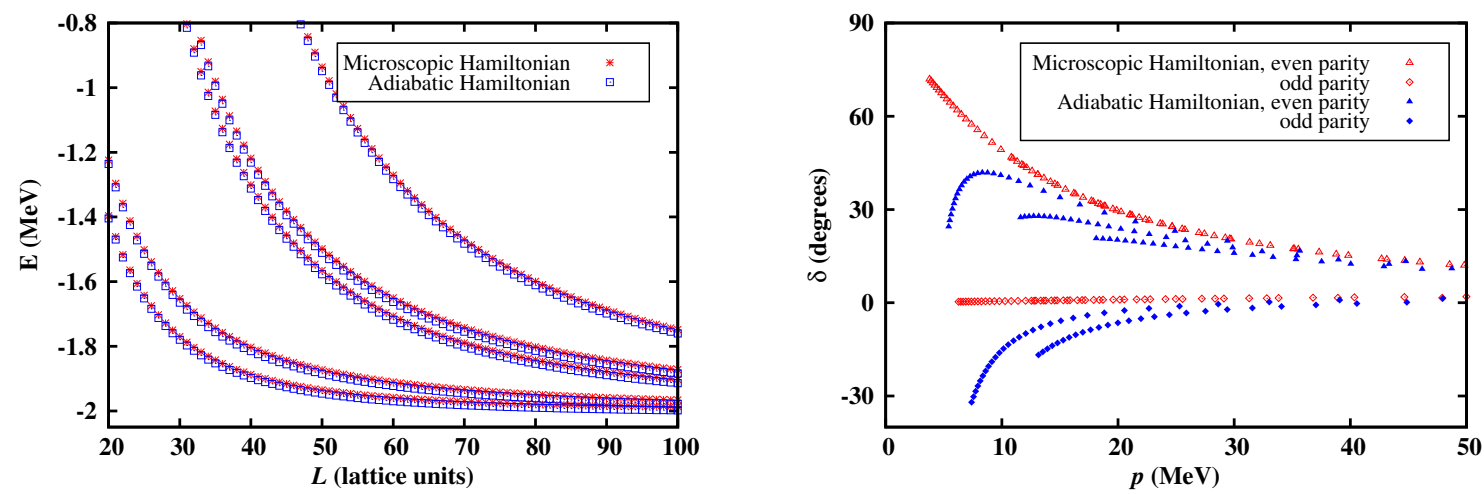

Figure 1: Left panel: Finite-volume energies extracted from the microscopic Hamiltonian and the twocluster adiabatic Hamiltonian for the particle-dimer system in one dimension. There are five energy levels $E_{1} \ldots E_{5}$. Three correspond to even and two to odd parity phase shifts.

Right panel: The particle-dimer scattering phase shifts calculated from the data in the left panel using Lüscher's method. Red (blue) symbols show the results corresponding to the original (adiabatic) Hamiltonian. For the adiabatic Hamiltonian small systematic errors in connection with Lüscher's formula lead to only an approximate separation of phase shifts with even and odd parity.

scattering phase shifts calculated by Lüscher's method. For this calculation we used the same finite-volume energies as in the left panel. Although there is no significant difference in the energy spectra of the original Hamiltonian and the adiabatic one, the resulting phase shifts show large discrepancies at low energies. Also, since low-energy phase shifts are extracted from boxes where the box sizes $L$ are very large and the level spacing between energy levels is small, tiny differences in energy levels are significant. This results in a more distinct disagreement at low energies if the phase shifts are derived using different energy levels of the adiabatic Hamiltonian. This example illustrates the sensivity of Lüscher's method in finite-volume energy calculations for low-energy nuclear scattering to small contaminations in energies.

Furthermore, the binding energies of the nuclei involved in the scattering process are different due to the finite volume $[13,14,15,16,17]$. In order to circumvent these problems, we introduce another approach for the second step of the adiabatic projection method. The main point is to directly analyze the cluster wave functions which are generated by the adiabatic Hamiltonian and extract the scattering information from their asymptotic part.

\section{Asymptotic cluster wave functions}

We need to explain why the cluster wave functions generated by the adiabatic Hamiltonian can be useful for phase shift extraction. First let us introduce a relative error tolerance $\varepsilon$. Let us also define a time scale $\tau_{\varepsilon}$ at which the initial cluster states are relaxed into a ground state with a relative contamination less than $\varepsilon$. This relative contamination happens due to excited cluster states. As we apply Euclidean time projection for the time duration $\tau_{\varepsilon}$, each cluster undergoes spatial diffusion. The average distance of this diffusion process is proportional to $\sqrt{\tau_{\varepsilon} / M}$. Thereby, $M$ is the mass of the corresponding cluster. This diffusion lengths $d_{\mathcal{E}, i}$ have to be much smaller than the box size $L$ and also the initial cluster separation $\vec{R}$ so that the dressed clusters $|\vec{R}\rangle_{\tau_{\varepsilon}}$ do not overlap. Let 
us define an asymptotic region $|\vec{R}|>R_{\varepsilon}$, where we call $R_{\varepsilon}$ the asymptotic radius, such that the overlap between the dressed clusters is less than the error scale $\varepsilon$. In this asymptotic region the dressed clusters are widely separated and only feel long-range forces. The effective Hamiltonian $H^{\text {eff }}$ for such system is then a free lattice Hamiltonian for two point particles accompanied by the long-range interactions inherited from the microscopic Hamiltonian. In the asymptotic region we can write

$$
\begin{aligned}
& {\left[N_{\tau}\right]_{\vec{R}, \vec{R}^{\prime}}=c\left[e^{-2 H^{\mathrm{eff}} \tau}\right]_{\vec{R}, \vec{R}^{\prime}},} \\
& {\left[H_{\tau}\right]_{\vec{R}, \vec{R}^{\prime}}=c\left[e^{-H^{\mathrm{eff}} \tau} H^{\mathrm{eff}} e^{-H^{\mathrm{eff}} \tau}\right]_{\vec{R}, \vec{R}^{\prime}},}
\end{aligned}
$$

where the coefficient $c$ is determined by the overlap of the initial single-cluster states (Eq. (2.1)) and the exact single-cluster energy eigenstates. Since

$$
\left[N_{\tau}^{-1 / 2}\right]_{\vec{R}, \vec{R}^{\prime}}=c^{-1 / 2}\left[e^{H^{\mathrm{eff}} \tau}\right]_{\vec{R}, \vec{R}^{\prime}},
$$

we conclude that in the asymptotic region the adiabatic and the effective cluster Hamiltonian coincide,

$$
\left[H_{\tau}^{a}\right]_{\vec{R}, \vec{R}^{\prime}}=\left[H^{\mathrm{eff}}\right]_{\vec{R}, \vec{R}^{\prime}} .
$$

This shows that in the asymptotic region the cluster wave functions correspond to the usual two-point particle wave functions. This allows the application of wave function techniques to them. In the following we use several variants of wave function techniques. Subsequently, we compare the resulting scattering information with those calculated by the Lüscher's energy spectrum method which employs the scattering state energy and not the wave function.

In the further analysis we use a lattice Hamiltonian that describes the interaction of three particle species. Let $b_{s}$ and $b_{s}^{\dagger}$ be the annihilation and creation operators for each particle species $s$. We use the Hamiltonian

$$
\begin{aligned}
H_{0} & =\sum_{s} \sum_{\hat{l}} \sum_{\vec{n}} \frac{1}{2 m_{s}} b_{s}^{\dagger}(\vec{n})\left[2 b_{s}(\vec{n})-b_{s}(\vec{n}+\hat{l})-b_{s}(\vec{n}-\hat{l})\right] \\
V & =\sum_{s<s^{\prime}} \sum_{\vec{n}} C_{S, s^{\prime}}: b_{s}^{\dagger}(\vec{n}) b_{s}(\vec{n}) b_{s^{\prime}}^{\dagger}(\vec{n}) b_{s^{\prime}}(\vec{n}):
\end{aligned}
$$

Here $\vec{n}(\hat{l})$ denotes lattice (unit) vectors along all possible axes in $D$ spatial dimensions. We tune the coefficients $C_{s, s^{\prime}}$ to produce a bound state of $E=-2.0000 \mathrm{MeV}(E=-2.2246 \mathrm{MeV})$ in $D=1$ $(D=3)$, see [12] for more details.

The easiest wave function method consists in fitting the asymptotic cluster wave function to its expected form. For a finite-range potential (no additional Coulomb interaction) the two cluster wave function in the asymptotic region $r>R_{\varepsilon}$ is given by

$$
\begin{aligned}
\Psi_{\ell}^{(p)}(r) & =A_{\ell} \cos \left(p r+\delta_{\ell}-\ell \pi / 2\right) & & \text { for one dimension, } \\
\Psi_{\ell, m_{\ell}}^{(p)}(\vec{r}) & =R_{\ell}^{(p)}(r) Y_{\ell, m_{\ell}}(\theta, \phi) & & \text { for three dimensions. }
\end{aligned}
$$



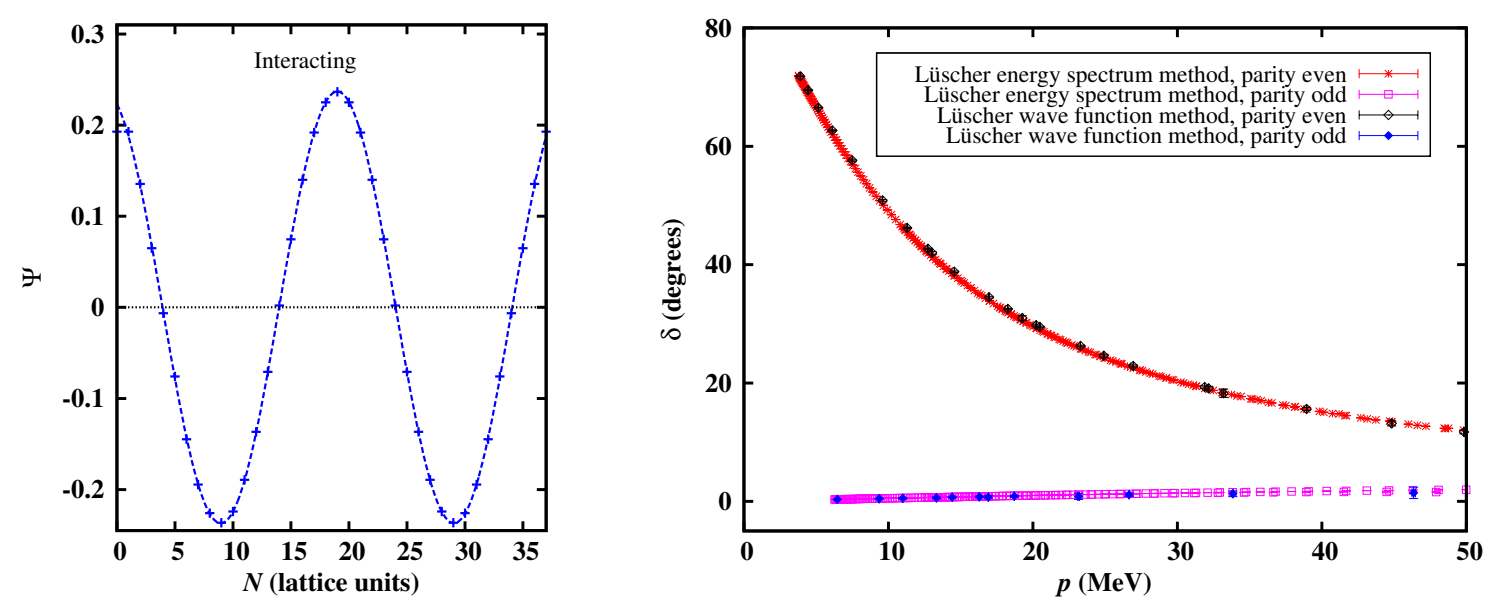

Figure 2: Particle-dimer phase shifts in one dimension calculated using the Lüscher wave function method. Left panel: Wave function lattice data (crosses) fitted to the asymptotic form (dashed line). Right panel: Comparison of the phase shifts calculated using the Lüscher periodic-box wave function method and Lüscher's finite-volume method with the exact energy spectrum.
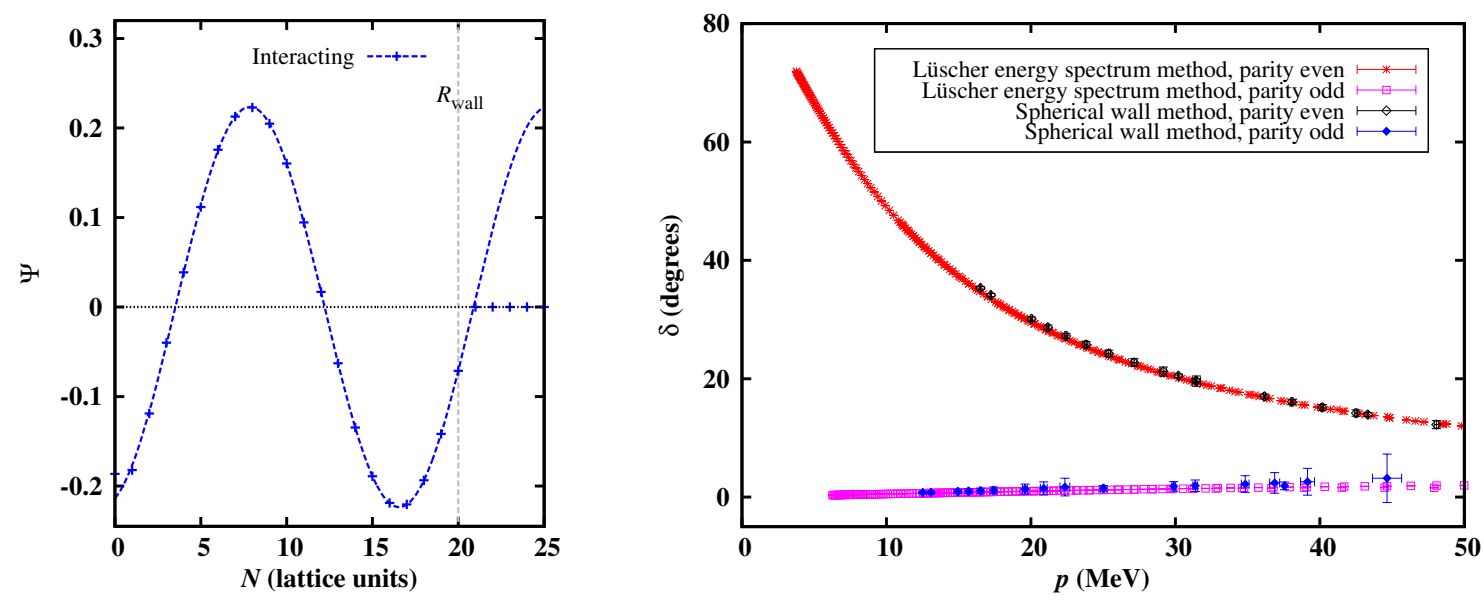

Figure 3: Particle-dimer phase shifts in one dimension calculated using the spherical-wall method in one dimension with a three-parameter fit. Left panel: Wave function lattice data (crosses) fitted to the asymptotic form (dashed line). Right panel: Comparison of the phase shifts calculated using the spherical wall method and Lüscher's finite-volume method with the exact energy spectrum.

For the one-dimensional case, there is no angular momentum. In this case we use the notation $\ell=0$ for even parity and $\ell=1$ for odd parity. In three dimensions in the case of a short range potential, the radial part $R_{\ell}^{(p)}(r)$ of the total wave function has the asymptotic form

$$
R_{\ell}^{(p)}(r)=A_{\ell}\left[\cos \delta_{\ell}(p) j_{\ell}(p r)-\sin \delta_{\ell}(p) n_{\ell}(p r)\right]
$$

The angular part of the total wave function is described by the spherical harmonics $Y_{\ell, m_{\ell}}(\theta, \phi)$. In the above equation $A_{\ell}$ is a normalization coefficient, $j_{\ell}$ is the spherical Bessel functions of the first kind and $n_{\ell}$ of the second. We refer to this method as the Lüscher wave function method. Fig. 2(a) shows the application for an example case in one dimension. The phase shift analysis 

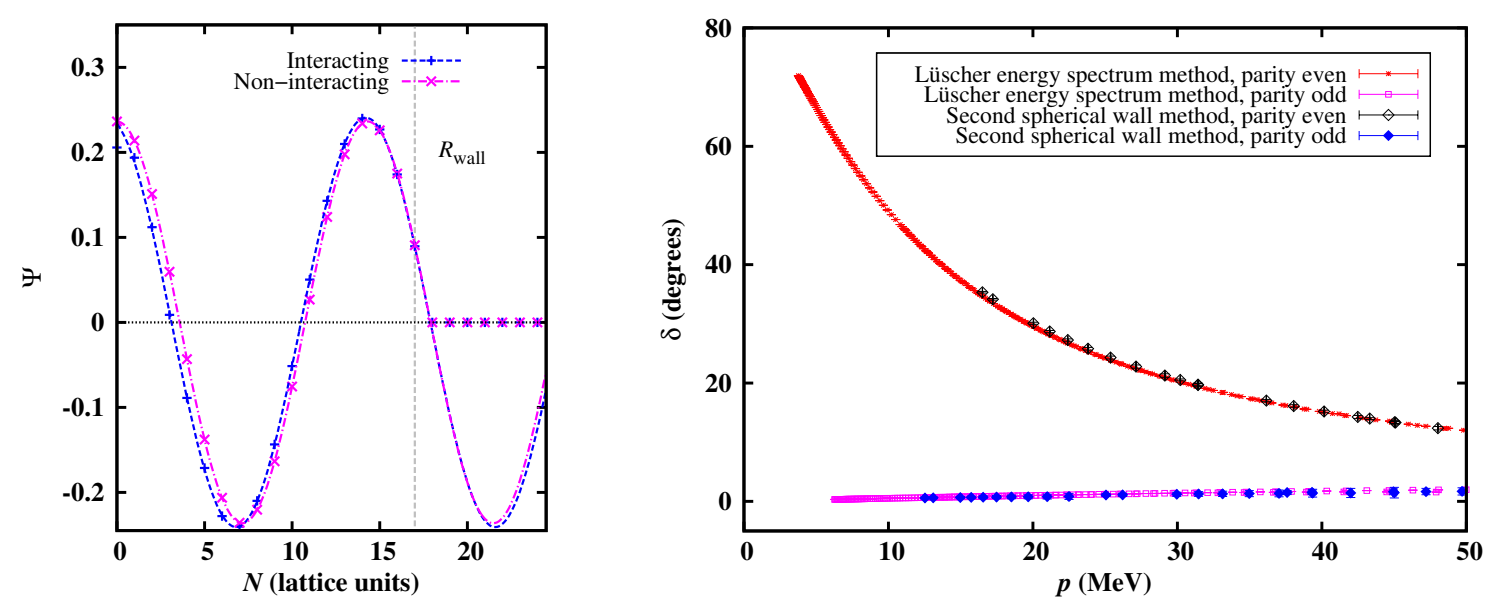

Figure 4: Particle-dimer phase shifts in one dimension using the second spherical wall approach with $R_{\text {wall }}^{\prime}$ determined from the non-interacting wave function. Left panel: Wave function lattice data (crosses) fitted to the asymptotic form (dashed line) for the interacting (blue) and non-interacting (magenta) case. Right panel: Comparison of the phase shifts calculated using the second spherical wall approach and Lüscher's finite-volume method with the exact energy spectrum.

was done for several lattices with $N=6 \ldots 100$ in lattice units. The Lüscher's energy spectrum method is applied to all of them and the Lüscher periodic-box wave function method to the lattices with $N=10,20, \ldots 100$ in lattice units. The results are shown in Fig. 2(b) to be in a very good agreement between both methods. This method is applicable in one dimension but is less useful in three dimensions, where the available computation resources would allow us to compute phase shifts only at large momenta.

The next method we apply is the spherical wall approach. The lattice version of this method of calculating phase shifts for point-like particles was introduced by Borasoy et al. [18]. As in the continuum version [19], the relative separation of the two particles or - as we consider twocluster systems - the relative separation between two clusters is confined by a wall boundary of radius $R_{\text {wall }}$. The standing wave functions in the box have the same expected asymptotic form described by the formula (3.8). This method allows for the calculation of several phase shift data points per one chosen lattice volume, since the variation of the wall radius, $R_{\text {wall }}$ changes the energy of the standing wave functions. This is especially important for calculations in three dimensions since, usually, only small volumes are available there. We distinguish between two versions of this method. The first one is a fit to three parameters: the overall normalization, momentum and phase shift of the interacting wave functions. Fig. 3 shows the result of this approach for the case $N=50$ and $R_{\text {wall }}=20$ in lattice units. The results for this method are also in very good agreement with Lüscher's energy spectrum method. The phase shifts are calculated for $N=50$ and $R_{\text {wall }}=13 \ldots 23$ in lattice units.

Another version of the spherical wall method is a two-parameter fit for the interacting wave function. The third parameter is obtained from the information of the the non-interacting particledimer wave function. The background for this is the following observation. The wave function on the lattice vanishes at a slightly larger radius than the one imposed on the lattice (in the given example in Fig. 4, the hard wall is set at $R_{\text {wall }}=17$, while the wave function is zero at $R_{\text {wall }}+\varepsilon=17.901$ ). 

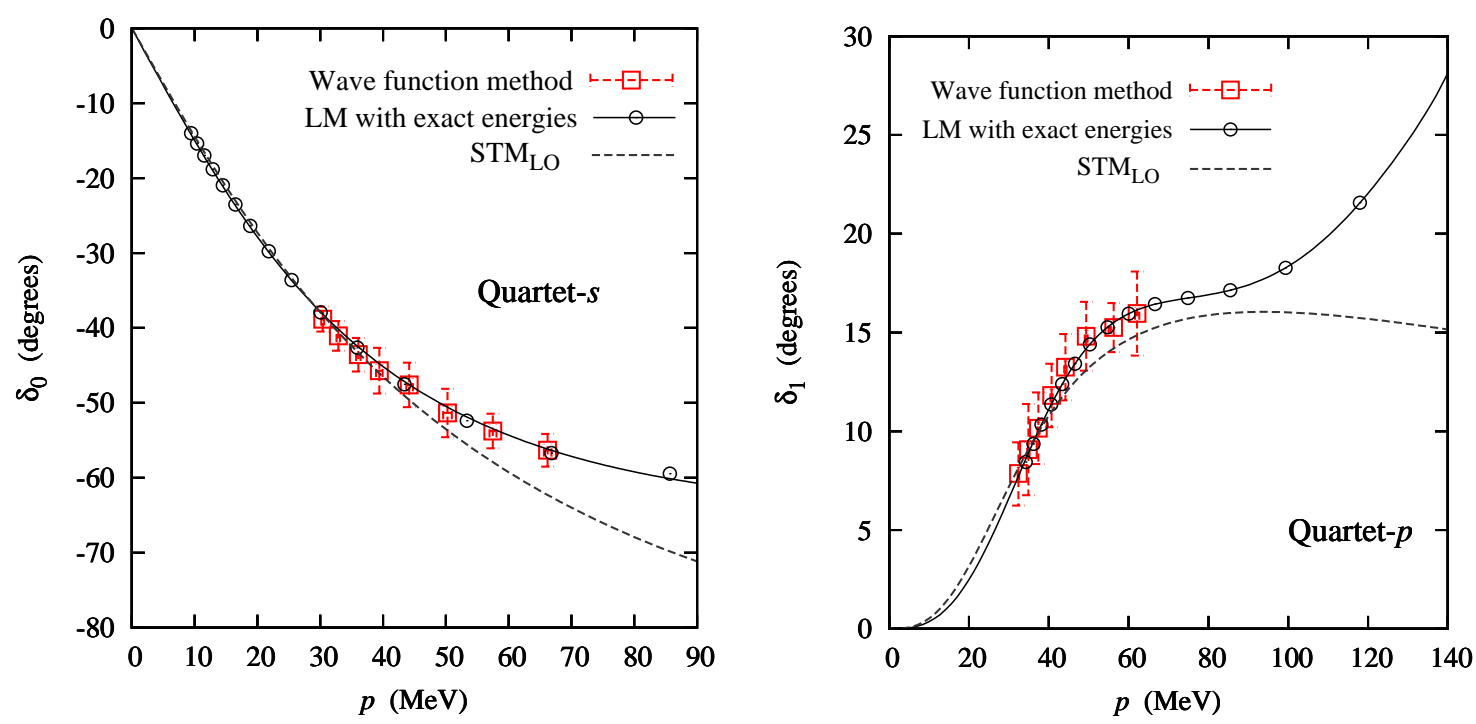

Figure 5: The $s$-wave (left panel) and $p$-wave (right panel) scattering phase shifts for fermion-dimer scattering in three dimensions. We compare phase shifts calculated using the the spherical wall approach with $R_{\text {wall }}^{\prime}$ determined from the non-interacting wave function with Lüscher's finite-volume method using the exact energy spectrum.

However, the difference in the value of $\varepsilon$ between the interacting and the non-interacting system is negligible. Therefore, we use the information from the non-interacting wave functions to fix $R_{\text {wall }}^{\prime}=R_{\text {wall }}+\varepsilon$ and have two free parameters $\left(p\right.$ and $\left.\delta_{l}\right)$ in the interacting system. The phase shifts shown in the right panel of Fig. 4 use the same data set as in Fig. 3, namely $N=50$ and $R_{\text {wall }}=13 \ldots 23$ in lattice units. Therefore, the results are basically the same as for the three parameter spherical wall approach, but have smaller confidence intervalls which is important in three dimensions.

For the three-dimensional fermion-dimer calculation, this method turned out to be the most promising one of the four possible approaches explored in the one dimensional system. Therefore the results in three dimensions are only shown for this method. In Fig. 5, we present the results for a three-dimensional fermion-dimer scattering process. The squares represent lattice results for the $s$-wave and $p$-wave scattering phase shifts using the adiabatic projection method with $\tau=$ 0.37 and the spherical wall method. They are compared with the exact lattice results obtained using Lüscher's method (circles). Hereby, Lüscher's method was applied to the energies of the microscopic Hamiltonian $H$. The lines correspond to continuum results obtained via the STM equation (dashed) and the fits of the data on the lattice to an effective range expansion (solid),

$$
p^{2 \ell+1} \cot \delta_{\ell}(p)=-\frac{1}{a_{\ell}}+\frac{1}{2} r_{\ell} p^{2}+\mathscr{O}\left(p^{4}\right)
$$

The last method is an attempt to calculate scattering information for very low energies using small volumes. This is a disadvantage of the spherical wall method since the radius of the wall has to at most half to the lattice size. This results in very small volumes in three dimensions. In order to probe for low energies we have to choose more complicated boundary conditions. Thus, 

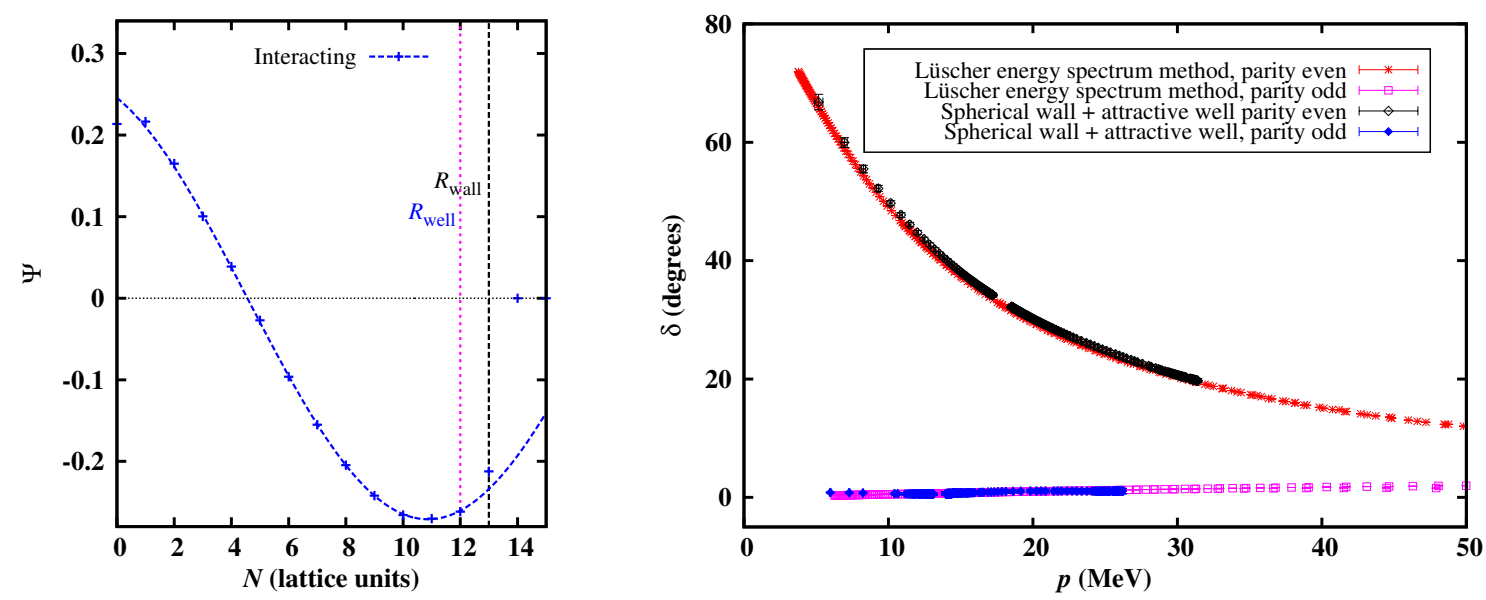

Figure 6: Particle-dimer phase shifts in one dimension with the spherical wall and an attractive well potential. Left panel: Wave function lattice data (crosses) fitted to the asymptotic form (dashed line). Right panel: Comparison of the phase shifts calculated using the approach based on the combination of the spherical wall and attractive well with Lüscher's finite-volume method using the exact energy spectrum.

we add an attractive well potential in front of the hard wall boundary. The depth of the well is an adjustable continuous parameter. The example shown in Fig. 6 corresponds to the case of $N=30$, $R_{\text {wall }}=13$ and $R_{\text {well }}=12$ in lattice units. The phase shifts shown in the right panel of Fig. 6 are calculated for $N=50, R_{\text {wall }}=23$ and different well depths. The agreement to the Lüscher's energy spectrum method - also for small energies - is very good. As the attractive potential becomes deeper the wave function corresponds to a wave function in an effectively larger box and through this to a wave function with smaller energy. However, the wave function is also getting distorted in the outer asymptotic region such that at some point the expected asymptotic form according to Eq. (3.7) is not a good fit function anymore.

\section{Summary}

In this report we argued that the adiabatic Hamiltonian reduces to a simple position-space free cluster Hamiltonian in the asymptotic region. This allows us to avoid the computation of the energy spectrum and use the Lüscher's method which is prone to potentially large errors. The phase shifts are calculated using the cluster wave function attained from the adiabatic Hamiltonian instead. We presented four different variations of the wave function techniques to calculate scattering information. The first one is a simple matching of the wave function to its expected form in the asymptotic region according to Eq. (3.7). The next two methods involve imposing a hard boundary on the relative separation of the clusters. The difference between the two is the use of the information from the non-interacting wave function. If this information is used to determine the radius where the wave function vanishes $R_{\text {wall }}^{\prime}$, the fit of the interacting wave function is more stable because of a reduced number of fitting parameters. The last method makes use of more complicated boundary conditions, namely an additional attractive well in front of the hard wall boundary. A great advantage of this method is that it allows to compute phase shifts for very low energies in small 
boxes. However, this additional potential also distorts the wave functions which is a disadvantage and results in potentially large confidence intervals.

\section{Acknowledgments}

This work was supported in part by the U.S. Department of Energy grant DE-FG02-03ER41260 (D.L. and M.P.), U.S. Department of Education GAANN Fellowship (M.P.), the Seventh Framework Programme of EU, the ERC project 259218 NUCLEAREFT (E.E. and A.R.) and the European Community-Research Infrastructure Integrating Activity Study of Strongly Interacting Matter (acronym HadronPhysics3, Grant Agreement n. 283286).

\section{References}

[1] K. Orginos, A. Parreno, M. J. Savage, S. R. Beane, E. Chang and W. Detmold, Two nucleon systems at $m_{\pi} \sim 450 \mathrm{MeV}$ from lattice QCD, Phys. Rev. D 92, no. 11, 114512 (2015) [arXiv:1508.07583 [hep-lat]].

[2] S. Elhatisari, D. Lee, G. Rupak, E. Epelbaum, H. Krebs, T. A. Lähde, T. Luu and U. G. MeiSSner, Ab initio alpha-alpha scattering, Nature 528, 111 (2015) [arXiv:1506.03513 [nucl-th]].

[3] E. Epelbaum, H. Krebs, D. Lee and U.-G. Meißner, Ab initio calculation of the Hoyle state, Phys. Rev. Lett. 106, 192501 (2011) [arXiv:1101.2547 [nucl-th]].

[4] E. Epelbaum, H. Krebs, T. A. Lähde, D. Lee and U.-G. Meißner, Structure and rotations of the Hoyle state, Phys. Rev. Lett. 109, 252501 (2012) [arXiv:1208.1328 [nucl-th]].

[5] T. A. Lähde, E. Epelbaum, H. Krebs, D. Lee, U.-G. Meißner and G. Rupak, Lattice Effective Field Theory for Medium-Mass Nuclei, Phys. Lett. B 732, 110 (2014) [arXiv:1311.0477 [nucl-th]].

[6] E. Epelbaum, H. Krebs, T. A. Lähde, D. Lee, U.-G. Meißner and G. Rupak, Ab Initio Calculation of the Spectrum and Structure of ${ }^{16} O$, Phys. Rev. Lett. 112, no. 10, 102501 (2014) [arXiv:1312.7703 [nucl-th]].

[7] G. Rupak and D. Lee, Radiative capture reactions in lattice effective field theory, Phys. Rev. Lett. 111, no. 3, 032502 (2013) [arXiv:1302.4158 [nucl-th]].

[8] M. Pine, D. Lee and G. Rupak, Adiabatic projection method for scattering and reactions on the lattice, Eur. Phys. J. A 49, 151 (2013) [arXiv:1309.2616 [nucl-th]].

[9] S. Elhatisari and D. Lee, Fermion-dimer scattering using an impurity lattice Monte Carlo approach and the adiabatic projection method, Phys. Rev. C 90, no. 6, 064001 (2014) [arXiv:1407.2784 [nucl-th]].

[10] M. Lüscher, Volume Dependence of the Energy Spectrum in Massive Quantum Field Theories. 2. Scattering States, Commun. Math. Phys. 105, 153 (1986).

[11] M. Lüscher, Two particle states on a torus and their relation to the scattering matrix, Nucl. Phys. B 354, 531 (1991).

[12] A. Rokash, M. Pine, S. Elhatisari, D. Lee, E. Epelbaum and H. Krebs, Scattering cluster wave functions on the lattice using the adiabatic projection method [arXiv:1505.02967 [nucl-th]].

[13] S. Bour, S. König, D. Lee, H.-W. Hammer and U.-G. Meißner, Topological phases for bound states moving in a finite volume, Phys. Rev. D 84, 091503 (2011) [arXiv:1107.1272 [nucl-th]]. 
[14] S. Bour, H.-W. Hammer, D. Lee and U.-G. Meißner, Benchmark calculations for elastic fermion-dimer scattering, Phys. Rev. C 86, 034003 (2012) [arXiv:1206.1765 [nucl-th]].

[15] M. Lüscher, Volume Dependence of the Energy Spectrum in Massive Quantum Field Theories. 1. Stable Particle States, Commun. Math. Phys. 104, 177 (1986).

[16] S. König, D. Lee and H.-W. Hammer, Volume Dependence of Bound States with Angular Momentum, Phys. Rev. Lett. 107, 112001 (2011) [arXiv:1103.4468 [hep-lat]].

[17] S. König, D. Lee and H.-W. Hammer, Non-relativistic bound states in a finite volume, Annals Phys. 327, 1450 (2012) [arXiv:1109.4577 [hep-lat]].

[18] B. Borasoy et al., Two-particle scattering on the lattice: Phase shifts, spin-orbit coupling, and mixing angles, Eur. Phys. J. A 34, 185 (2007) [arXiv:0708.1780 [nucl-th]].

[19] J. Carlson, V. R. Pandharipande and R. B. Wiringa, Variational calculations of resonant states in ${ }^{4} H e$, Nucl. Phys. A 424, 47 (1984). 